\title{
Les traces écrites scolaires : une cristallisation de discours
}

Written traces in the classroom: a cristallization of discourse

\section{Aurore Promonet}

\section{(2) OpenEdition}

1 Journals

Édition électronique

URL : http://journals.openedition.org/aad/2465

DOI : $10.4000 /$ aad. 2465

ISSN : $1565-8961$

Éditeur

Université de Tel-Aviv

Référence électronique

Aurore Promonet, "Les traces écrites scolaires : une cristallisation de discours », Argumentation et Analyse du Discours [En ligne], 19 | 2017, mis en ligne le 15 octobre 2017, consulté le 23 septembre 2019. URL : http://journals.openedition.org/aad/2465; DOI : 10.4000/aad.2465

Ce document a été généré automatiquement le 23 septembre 2019.

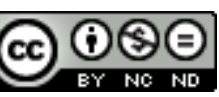

Argumentation \& analyse du discours est mis à disposition selon les termes de la licence Creative Commons Attribution - Pas d'Utilisation Commerciale - Pas de Modification 4.0 International. 


\title{
Les traces écrites scolaires : une cristallisation de discours
}

Written traces in the classroom: a cristallization of discourse

\author{
Aurore Promonet
}

\section{Introduction}

1 Nous souhaitons montrer comment le recours à l'analyse du discours permet de mettre en évidence la complexité d'un type d'écrit scolaire, appelé "trace écrite», qui s'élabore dans l'espace socio-discursif de la classe. Cette trace écrite est un écrit consigné dans les cahiers des élèves sous la direction de l'enseignant. Elle rend compte, dans l'après-coup, du travail que le groupe a accompli dans l'espace-temps de la classe. Après avoir décrit la trace écrite comme prescription institutionnelle et comme processus oralographique à travers un exemple, nous mettrons en œuvre les concepts qui nous permettent de l'envisager comme manifestation dialogique et interdiscursive. Enfin, nous tenterons de mettre en évidence les caractéristiques auctoriales de ce type d'écrit scolaire.

\section{La trace écrite comme prescription institutionnelle et didactique et comme processus oralographique}

\subsection{Les premières apparitions dans la littérature institutionnelle de l'expression « trace écrite »}

Les programmes scolaires et documents d'accompagnement concernant l'enseignement de l'histoire et de la géographie au collège sont les premiers à employer le terme de « trace » pour prescrire les usages du manuel et du cahier, qualifiés d'« outils du travail des élèves » (Programme du cycle central, sè et 4è, 1997 : 37). On peut lire : 
[...] il faut que le cahier personnel porte la trace des différents exercices effectués. [...] le cahier deviendra un véritable recueil personnel, témoin des difficultés et des erreurs mais aussi des progrès de l'élève. Complément personnalisé d'un manuel aux attributs restaurés, il trouverait, sous le contrôle du professeur, une forme plus conforme aux ambitions formatrices de l'enseignement de l'histoire et de la géographie. (Accompagnement des programmes de 3è, Histoire-géographie, 1999 : 15).

Ce paragraphe prescriptif pose la problématique de la trace écrite scolaire, telle qu'elle sera formulée dans la littérature institutionnelle puis dans la littérature scientifique. Il distingue le cahier de l'élève du manuel scolaire en soulignant le caractère personnel du cahier. Dans le même mouvement, il situe le cahier à l'interface entre l'enseignement et l'apprentissage dans la mesure où il le désigne comme un outil d'élève placé sous le contrôle de l'enseignant.

4 L'expression "trace écrite » est encore employée dans un rapport de l'Inspection générale de mathématiques intitulé Les traces écrites des élèves en mathématiques (Roux et Olivier, 2001). Les auteurs de ce rapport soulignent également la double appartenance $\mathrm{du}$ cahier dont l'organisation est décidée par l'enseignant mais dont le contenu reflète le travail des élèves.

5 En 2008, "l'élaboration d'une trace écrite» est explicitement prescrite dans le programme scolaire de français pour la classe de 6è (première année de collège) : «Le professeur initie progressivement l'élève à l'élaboration d'une trace écrite: préparation, synthèse ou réinvestissement du travail effectué en classe » (B.O. $\mathrm{n}^{\circ} 6 \mathrm{du}$ 28-08-08: 8). Cette prescription confirme que l'institution pense cet écrit scolaire comme un écrit qui se co-construit entre l'enseignant et les élèves, comme le fruit d'une écriture conçue par l'enseignant pour faire apprendre les élèves.

6 La recherche en éducation s'est intéressée à cet écrit scolaire dans le contexte des disciplines que nous venons de citer : en français (Nonnon 2004, Pottier 2005, Promonet 2015 b), en histoire-géographie (Meunier et Sala 2016), en mathématiques (Blochs 2009, Priolet 2013) et dans une perspective interdisciplinaire croisant la géographie, les sciences de la vie et de la terre et le français (Philippot et Niclot 2009). Ces travaux ont exploré la trace écrite dans une perspective didactique pour tenter d'en comprendre le fonctionnement et les usages en articulant activité des enseignants et apprentissage des élèves. Ils la définissent comme ce qui reste lorsque la séance se termine et que les élèves quittent la salle de classe. En dépit de la restitution lacunaire de la séance, elle a la fonction, pour les élèves et leur enseignant, de déclencheur de mémoire. Pour les lecteurs extérieurs (familles et corps d'inspection, notamment), elle constitue la preuve matérielle que quelque chose a eu lieu.

7 C'est ici selon une approche discursive que nous souhaitons aborder la trace écrite scolaire pour montrer en quoi elle participe de l'interdiscours scolaire. Nous partons du principe que cet écrit, circulant et faisant circuler au-delà de la classe les savoirs qui y sont abordés, cristallise un discours de l'École et un discours sur l'École. La trace écrite présente en effet les traits sémantiques de la cristallisation au sens de " concrétisation » (employé en chimie, le verbe « cristalliser " $^{1}$ signifie «transformer en cristaux » et dans un sens figuré, il signifie «fixer, concrétiser, rendre cohérent, sensible »). À l'issue de la séance, la trace écrite témoigne, dans une forme stabilisée, de ce qui s'est joué dans la classe, de l'organisation et de la cohérence de l'activité scolaire orale et écrite et, pour la rendre communicable, elle l'objective à travers la mise en 
mots de l'expérience vécue. Cependant, elle ne rend pas compte de son mode d'élaboration.

\subsection{Phases du processus d'écriture et éléments de la trace écrite scolaire}

8 Comme on l'a vu, dès son émergence dans les textes prescriptifs, la notion de «trace écrite scolaire » se caractérise par une indétermination entre trace d'apprentissage et trace d'enseignement. Illustrons cette affirmation par un exemple.

9 La trace écrite que nous présentons est conduite à l'école primaire et elle est extraite d'un corpus de transcriptions de séances de lecture menées dans douze classes de dernière année d'école primaire et de première année d'enseignement secondaire (Promonet, 2015b). Cette trace écrite présente trois éléments : la définition du genre du calligramme, deux calligrammes d'Apollinaire lus et étudiés dans la séance, et un calligramme inventé par l'élève. La confrontation avec la séance filmée permet de reconstituer les étapes de la genèse de cet écrit final; elle confirme ce que l'écrit suggère mais que seuls les élèves, leur enseignant et des observateurs sont en mesure d'articuler à l'activité effective qui en est à l'origine. Elle permet d'observer le tissage entre discours oraux et discours écrits dont résulte la trace écrite, et de distinguer l'interférence de trois sources énonciatives: la préparation - en amont - de l'enseignant, les interactions entre pairs, et les régulations professorales face aux réactions des élèves. La trace écrite se faisant l'écho de ce maillage oral/écrit, elle relève tout autant de l'ordre de l'oralité que de l'ordre de la scripturalité (Peytard 1970), à ceci près que l'énonciation orale, alors qu'elle influence le contenu et le rythme de l'élaboration de l'écrit, ne transparait pas dans les cahiers des élèves.

10 Une phase initiale de découverte, de déchiffrage et de compréhension du texte-support en nourrit pourtant l'élaboration : l'enseignant a copié un calligramme au tableau sous le regard de ses élèves. L'opération qui est alors engagée est un travail de reformulation (Promonet, 2015a) par linéarisation du texte selon le dispositif oralo-graphique (Bouchard et Parpette 2012) d'une dictée à l'adulte ${ }^{2}$. La mise en commun des propositions de lecture des élèves suppose en effet des échanges oraux jusqu'à validation collective. En prolongement de cette phase de découverte lors de laquelle ont été observés l'organisation textuelle particulière, la cohérence et la dimension esthétique du calligramme, les élèves découvrent deux autres calligrammes qu'ils linéarisent individuellement avant un retour collectif. La seule lecture des deux calligrammes de Guillaume Apollinaire qui figureront finalement dans la trace écrite sera susceptible d'engendrer le souvenir du travail de manipulation (déconstruction/ reconstruction) dont ils ont été l'objet.

11 Alors qu'elle n'a été élaborée que lors d'une deuxième phase de la séance, c'est la définition du genre poétique étudié qui apparait dans la première partie de la page. Cette définition puise à trois sources: un article de dictionnaire qui fournit une définition stabilisée mais dont seul l'enseignant dispose, des définitions tâtonnantes d'élèves découvrant le calligramme et les discussions au sein de la communauté de la classe, arbitrées par l'enseignant. Les élèves peuvent voir dans cette trace écrite le résultat d'une activité collégiale argumentée, négociée sur le plan sémantique, sur le plan lexical et sur le plan syntaxique. L'enseignant y retrouve, pour sa part, également son choix de pilotage de la séance, déterminé par le souci de valoriser le travail de ses 
élèves, leurs connaissances et leurs compétences langagières, méta-langagières et rédactionnelles.

Enfin, le troisième élément constitutif de cette trace écrite renvoie à un travail d'écriture d'invention qui correspond à la troisième phase de son élaboration. Ici, l'enseignant dirige une activité rédactionnelle qui place les élèves en situation de mobiliser des connaissances en cours de construction pour en stabiliser la maîtrise. Il leur fait verbaliser les étapes de construction d'un calligramme, il aide à la recherche d'idées, il étaye la phase de planification du texte à inventer au moyen de notations au tableau qui serviront de guide aux élèves pour la mise en ordre de leurs idées. Cette phase ne figure pas davantage dans la trace écrite finalement publiée dans les cahiers.

\section{Organisation méthodologique de la recherche}

La trace écrite détaillée ici doit être considérée dans sa singularité. Elle appartient à un corpus varié : les séances que nous avons étudiées ont donné lieu, selon les cas, à une activité centrée sur l'entrainement à la lecture orale fluide ou bien sur le travail de la compréhension en lecture, avec mise en place de stratégies de compréhension ou encore d'activités de lecture interprétative de textes littéraires. Face à la variété des données, des ajustements méthodologiques s'imposent. Notre corpus peut se prêter à une exploitation didactique : on peut y voir une trace de l'activité scolaire et l'étudier pour documenter l'activité de l'enseignant, l'activité de l'élève ou encore leur activité conjointe. Nous faisons ici le choix de lire nos données dans la perspective de leur fonctionnement discursif comme traces d'une activité de co-construction dialogique et interdiscusive. Notre étude ne se centre pas sur l'interlocution dans la classe mais exclusivement sur les modalités d'émergence d'un discours et la place faite aux énonciateurs de la classe. Dans ce but, nous avons reconstitué la genèse des traces écrites recueillies dans douze classes, sur le modèle des dossiers de genèse littéraires (De Biasi 2011) et scolaires (Doquet 2011). Commençons par présenter l'outillage méthodologique que nous avons mobilisé pour mettre en évidence ce que nous avons appelé la cristallisation du discours scolaire dans la trace écrite.

\subsection{Constitution d'un dossier de genèse}

Pour comprendre la composition des traces écrites recueillies dans les classes observées, nous avons procédé à l'enregistrement vidéo des douze séances. Nous avons recueilli la photographie de deux ou trois traces écrites par séance, sur les recommandations des enseignants. Avec leur autorisation, nous avons également recueilli les documents utilisés pour concevoir et piloter la séance: ouvrages de référence, manuel scolaire, écrits de préparation manuscrits ou saisis informatiquement. Chacun d'eux a disposé du film de sa séance et a eu la possibilité de le visionner en amont d'un entretien d'auto-confrontation simple, intégralement enregistré, dont le déroulement suit les options méthodologiques de la clinique de l'activité. Le chercheur visionne le film en compagnie de l'enseignant qui l'aide à appréhender ses prises de décision car la conduite de la trace écrite prend des formes que l'observateur ne peut interpréter: une hésitation, un changement de cap, un renoncement décidés dans la fulgurance du pilotage de la séance. Ce dispositif vise la compréhension fine du vouloir-faire et du vouloir-écrire de l'enseignant. En effet, 
[d]ans la situation d'autoconfrontation simple, à l'activité de l'opérateur qui dit ce qu'il fait ou ce qu'il aurait pu ou ne pas faire en se voyant à l'écran, répond l'activité $\mathrm{du}$ chercheur qui, voulant s'assurer une bonne compréhension, en est réduit aux conjectures qui n'ont, au mieux, qu'une valeur heuristique pour conduire l'entretien (Clot, Faïta, Fernandez et Scheller $2000: 5$ ).

\subsection{Le traitement des données}

Les traces écrites sont diverses sur le plan de leur structuration sémiotique mais elles présentent des caractéristiques communes: intégralement saisies au clavier ou entièrement manuscrites, toute distinction entre les contributeurs du discours produit, entre apport enseignant et apport apprenant est gommée; composites, elles se constituent dans un processus de mise en forme textuelle d'éléments distincts, par assemblage, synthèse et organisation de fragments choisis.

Nous transposons les méthodes d'analyse génétique à l'écriture scolaire de la trace écrite ${ }^{3}$ : nous l'avons considérée comme un texte publié, dont l'avant-texte documenterait la naissance (Bellemin-Noël 1972 : 15). Les documents préparatoires, les interactions orales de la classe comme les inscriptions au tableau constituent cet avanttexte et sont autant d'indices des états successifs qui précèdent la forme ultime, consignée dans les cahiers, stabilisée pour publication. On est à même d'y repérer les grandes phases d'écriture correspondant aux différentes situations discursives. Les enregistrements vidéo et audio, comme les traces écrites photographiées dans les cahiers et au tableau, sont transcrits intégralement dans le respect de leur organisation (spatialisation, codes couleur, distinction de tailles de caractères signalant titres et intertitres, etc.).

17 Nous avons considéré, à la suite de De Biasi (2011) que dans l'espace-temps de la séance se succèdent une phase rédactionnelle (structuration de la trace écrite), une phase prééditoriale (formalisation au tableau) et une phase éditoriale (copie, consignation dans les cahiers, valant publication de l'écrit produit). Mettre ces phases en évidence permet de montrer comment la trace écrite, parce qu'elle est prescrite, s'impose à la fois comme moteur de l'activité scolaire et comme résultat de l'activité de lecture. La phase pré-rédactionnelle précède l'ouverture de la séance et se matérialise en écrits préparatoires ; les phases rédactionnelle, pré-rédactionnelle et éditoriale, quant à elles, s'inscrivent dans des correspondances plus aléatoires, avec des phases orales, écrites, oralo-graphiques et elles se matérialisent en énoncés écrits et fixés sur des supports variés (cahiers, tableau, feuilles libres). Ces énoncés sont plus ou moins pérennes : les brouillons et écrits d'essai et de recherche, très liés à leur contexte d'émergence, sont moins mobilisables dans des taches scolaires ultérieures que des définitions qui sont avantage décontextualisées et qui relèvent d'un discours second. 


\section{Détermination des dimensions discursives de la trace écrite scolaire}

\subsection{Structuration de la trace : de l'énonciation à la production de discours}

La constitution d'un avant-texte facilite la description de la dimension interdiscursive de cet écrit scolaire dont la variété sémiotique ne reflète pas la variété énonciative (ainsi, par exemple, la partition entre part manuscrite et part dactylographiée ne recouvre pas exactement l'écriture apprenante et l'écriture enseignante).

La trace écrite se définit comme une unité d'échange verbal : l'organisation spatiale et textuelle de certaines traces écrites renvoie à l'alternance dialogale des questions/ réponses et des interventions enseignant/élèves. Entre prescriptions en amont et attentes de lecteurs en aval, la trace écrite s'élabore et se finalise dans un contexte dialogique à plusieurs niveaux: au fil des échanges, il se manifeste dans des négociations référées à des discours variés (programmes scolaires, formation professionnelle initiale et continue des enseignants, définitions de dictionnaire, théories didactiques, théories littéraires...).

La trace écrite ne vaut pas pour elle-même: elle répond à des prescriptions pédagogiques et didactiques. Cette scène discursive englobante brouille les voix : les attentes d'un lectorat attentif orientent d'avance l'interprétation des échanges verbaux et placent l'enseignant dans une "attitude responsive active » et elle lui impose de tenir compte des «harmoniques dialogiques" (Bakhtine 1979: 274 et 300) propres à l'institution. L'espace interdiscursif dont participe la trace écrite suppose en effet l'existence de discours passés et à venir: traces écrites déjà produites, discours prescriptifs pré-existants, commentaires et critiques.

\subsection{Trace écrite et auctorialité}

21 L'analyse génétique de la trace écrite met en évidence la liberté rédactionnelle et éditoriale des élèves. Elle atteste d'une volonté enseignante de valoriser leur « ethos discursif » (Amossy 2002 : 239). Ainsi, une enseignante d'école primaire, confrontée à l'enregistrement d'une séance de lecture littéraire, revendique une stratégie de coédition lors de l'entretien d'auto-confrontation: elle place ses élèves en situation de faire œuvre, en dépit de l'absence de signature :

C'est eux qui décident d'écrire en vert, d'écrire en bleu, d'écrire à tel niveau, de positionner, de faire une frise. J'ai réfléchi avec eux quand même. J'ai mes idées à la maison. Mais j'aime bien que les affichages soient aussi créés par les élèves pour qu'ils se les approprient.

Au service de l'ethos des élèves (Bucheton et Soulé 2009), la cristallisation oralographique propre à la trace écrite ne se réduit pas à une activité de surface, même si l'analyse discursive de la trace produite désigne l'activité de l'élève davantage comme une activité lectorale qu'auctoriale. Son statut de débutant et d'apprenant détermine sa contribution au discours scolaire.

Les analyses didactiques confortent cette approche, dans la mesure où elles mettent en évidence l'enjeu de la séance et reconnaissent un rôle actif à l'élève. Elles soulignent l'importance de la fonction d'institutionnalisation de la trace écrite (Brousseau 1988, 
Reuter, Cohen-Azria, Daunay, Delcambre et Lahanier-Reuter 2013). Néanmoins, si l'élève prend part au discours, il est co-énonciateur mais pas auteur.

La figure de l'auteur est discrète dans les traces écrites observées mais les catégories développées par Maingueneau (2009) nous permettent de définir le statut auctorial de l'enseignant. Il est auteur-répondant de la trace écrite : il répond de ce qui se publie dans les cahiers, il en contrôle le contenu et la forme en tant que passeur du discours de l'enseignement.

Il est auteur-acteur dans la mesure où son activité professionnelle s'organise autour de l'écriture de la trace écrite : il la compose ou la prépare avant la séance parce que sa profession d'enseignant l'exige ; il conjugue formation professionnelle et programmes scolaires pour cadrer son activité d'enseignement et l'activité d'apprentissage de ses élèves. Cependant, il n'assume pas le statut d'auteur-auctor puisqu'il n'est pas un professionnel de l'écriture. Si l'enseignant exerce son autorité dans la classe, il ne la revendique pas dans la trace écrite. L'énoncé produit est lissé par l'effacement énonciatif de rigueur. Du fait de l'absence d'indices de l'énonciation, la trace écrite semble s'énoncer elle-même, elle se présente comme un énoncé non pris en charge.

L'exemple de la séance d'étude des calligrammes illustre la réalité de la classe et la prégnance d'une communauté scolaire élargie; elle montre une auctorialité caractérisée par le triple contexte d'émergence qui nourrit et contraint son élaboration au niveau de l'activité professionnelle d'enseignement, au niveau de l'activité didactique et au niveau de l'activité scolaire d'apprentissage.

\section{Conclusion}

L'étude génétique de la trace écrite s'apparente métaphoriquement à une étude archéologique.

Elle montre que, par essence, cet écrit scolaire ne peut être ni le texte des élèves ni celui de l'enseignant. La trace écrite est un écrit sans auteur désigné. Elle contient sous forme condensée et cristallisée la combinaison des discours enseignant et apprenant, cadrés par des prescriptions institutionnelles et scientifiques mais aussi par les attentes des usagers de l'institution scolaire qu'il s'agit d'anticiper. Cependant l'enseignant conduit la trace écrite. Il adapte doublement cet écrit composite à une situation immédiate et seconde. Il harmonise des pratiques discursives parfois peu compatibles, il orchestre des voix hétérogènes.

\section{BIBLIOGRAPHIE}

Amossy, Ruth. 2002. « Ethos », Charaudeau, Patrick \& Dominique Maingueneau (éds), Dictionnaire d'analyse du discours (Paris : Seuil)

Bakhtine, Mikhaïl. 1979. Esthétique de la création verbale (Paris : Gallimard) 
Bellemin-Noël, Jean. 1972. Le texte et l'avant-texte (Paris : Librairie Larousse)

Blochs, Bernard. 2009. La place du cahier de cours dans les apprentissages mathématiques en classe de 4è. Pratiques et conceptions de professeurs et d'élèves. (Thèse de doctorat, Université Paris-Diderot Paris VII), en ligne : https://halshs.archives-ouvertes.fr/tel-00437058/

Bouchard, Robert \& Chantal Parpette. 2012. «Littéracie universitaire et oralographisme : le cours magistral entre écrit et oral », Pratiques 153-154, Littéracies universitaires : nouvelles perspectives. $195-210$

Brousseau, Guy. 1988. «Les différents rôles du maitre ». Bulletin de l'A.M.Q. Montréal, 14-24, en ligne : https://hal.archives-ouvertes.fr/hal-00497481

Bucheton, Dominique \& Yves Soulé. 2009. « Les gestes professionnels et le jeu des postures de l'enseignant dans la classe : un multi-agenda de préoccupations enchâssées ", Education et Didactique 3, 29-48

Charaudeau, Patrick \& Dominique Maingueneau. 2002. Dictionnaire d'analyse du discours (Paris : Seuil)

Clot, Yves, Daniel Faïta, Gabriel Fernandez \& Livia Scheller. 2000. « Entretiens d'autoconfrontation croisée : une méthode en clinique de l'activité ». Perspectives interdisciplinaires sur le travail et la santé 2-1. URL : http://pistes.revues.org/3833

De Biasi, Pierre-Marie. 2011. La génétique des textes (Paris : Nathan)

Doquet, Claire. 2011. L'Ecriture débutante - Pratiques scripturales à l'école élémentaire. (Rennes : P. U. de Rennes)

Maingueneau, Dominique. 2009. « Auteur et image d'auteur en analyse du discours » Argumentation et analyse du discours 3, https://aad.revues.org/660

Meunier, Christophe \& Céline Sala. 2016. « Enseigner l'histoire-géographie à l'école » (Malakoff : Dunod)

Nonnon, Elisabeth. 2004. «Travail visible et invisible : la trace écrite au tableau » Recherches 41, $17-30$

Peytard, Jean. 1970. «Oral et scriptural : deux ordres de situations et de descriptions linguistiques ", Langue française 6, 35-47

Philippot, Thierry \& Daniel Niclot. 2011. Analyser le travail des professeurs de collège à partir des "traces écrites » réalisées en classe. Communication présentée au congrès « Le travail enseignant au XXIe siècle ». INRP, Lyon

Pottier, Jean-Michel. 2005. « Trace écrite, trace écran. Génétique textuelle et pratiques scolaires », Pottier, Jean-Michel (éds). « Seules les traces font rêver». Enseignement de la littérature et génétique textuelle. Actes des cinquièmes rencontres des chercheurs en didactique littéraire. Reims, 1er et 2 avril 2004 (Reims : CRDP de Champagne-Ardenne)

Priolet, Maryvonne. 2013. «Place et rôle des traces écrites en résolution de problèmes numériques à l'école élémentaire : quelles méthodologies de recherche ? ", Actes du Congrès International de l'AECSE : AREF 2013 Actualité de la Recherche en Éducation et Formation (France : Montpellier) URL : http://www.aref2013.univ-montp2.fr/cod6/?q=content/3373-placeet-rôle-des-traces-écrites-en-résolution-de-problèmes-numériques-à-l'école-élémen

Promonet, Aurore. 2015a. « L'élaboration de la trace écrite : le rôle de la reformulation », Recherches 62, 31-50 
Promonet, Aurore. 2015b. Du cahier de l'élève à l'activité enseignante en classe de français. Étude de la trace écrite d'une séance de lecture, du cycle 3 à la 6è. (Thèse de doctorat, Université de Reims Champagne Ardenne), en ligne : http://www.theses.fr/2015REIML008

Reuter, Yves, Cora Cohen-Azria, Isabelle Delcambre \& Dominique Lahanier-Reuter. 2013. Dictionnaire des concepts fondamentaux des didactiques (Bruxelles : De Boeck)

Roux, Dominique \& Yves Olivier. 2001. Rapport de l'Inspection Générale de mathématiques : les traces écrites des élèves en mathématiques, en ligne : http://media.education.gouv.fr/file/86/3/6863.pdf

\section{NOTES}

1. «Cristallisation » (s.d.), Trésor de la langue française informatisé.

2. Ce dispositif est couramment utilisé à l'école primaire : l'enseignant prend en charge la dimension orthographique d'un texte collectivement composé par formulation orale en vue de sa consignation en langage écrit.

3. Une grille d'analyse permet de d'établir une correspondance entre phases scénariques et écriture de chaque trace écrite.

\section{RÉSUMÉS}

Nous analysons l'élaboration de la trace écrite du travail de la classe, en français, en séance de lecture, à partir de l'observation de classes de dernière année d'école primaire et de première année d'enseignement secondaire. Cette trace du travail de la classe est consignée dans les cahiers ou les classeurs des élèves, sous la conduite de l'enseignant. Coproduite par les acteurs de la classe, elle se constitue en texte du savoir scolaire dans la communauté de la classe durant la séance qui l'a fait naitre et au-delà. En effet, cet écrit référant à différentes activités et résultant de nombreux échanges cristallise un discours scolaire qui le dépasse largement. Notre projet consiste à aborder cette trace écrite selon l'approche de l'analyse du discours, dans ses dimensions dialogiques et interdiscursives.

We analyse school written traces from French lesson throught its production. In this purpose, we observed reading lessons at school and middle school (year 6 and 7 in UK). This written trace of the work of the class is deposited in pupils' exercise books under the direction of the teacher. This track is coproduced by the actors in the class. It's created from the knowedge of the class during the session and beyond. Indeed, this paper referring to various activities and resulting from lots of sparring matches, it crystallizes a school speech which exceeds it widely. Our goal is to approach this written trace by the discourse analysis, on its dialogical and interdiscursive characteristics.

\section{INDEX}

Mots-clés : analyse du discours, auctorialité, trace écrite

Keywords : authorship, discourse analysis, written trace 
AUTEUR

AURORE PROMONET

Université de Lorraine, Laboratoire CREM - EA 3476 